\title{
BMJ Open Prostate cancer with bone metastasis in Beijing: an observational study of prevalence, hospital visits and treatment costs using data from an administrative claims database
}

To cite: Zhuo L, Cheng Y, Pan Y, et al. Prostate cancer with bone metastasis in Beijing an observational study of prevalence, hospital visits and treatment costs using data from an administrative claims database. BMJ Open 2019;9:e028214. doi:10.1136/ bmjopen-2018-028214

- Prepublication history for this paper is available online. To view these files, please visit the journal online (http://dx.doi. org/10.1136/bmjopen-2018028214).

Received 28 November 2018

Revised 8 April 2019

Accepted 10 May 2019

Check for updates

(c) Author(s) (or their employer(s)) 2019. Re-use permitted under CC BY-NC. No commercial re-use. See rights and permissions. Published by BMJ.

For numbered affiliations see end of article.

\section{Correspondence to}

Dr Siyan Zhan;

siyan-zhan@bjmu.edu.cn and

Jian Lu;

lujian@bjmu.edu.cn

\section{ABSTRACT}

Objectives To estimate the prevalence of prostate cancer with bone metastasis in Beijing, and to estimate hospital visits and direct treatment costs among male urban employees with the disease in Beijing.

Design Cross-sectional observational study. Setting and participants Patients with prostate cancer and bone metastasis from the Urban Employee Basic Medical Insurance database covering the employed population of Beijing, China, from 2011 to 2014.

Primary and secondary outcome measures Prevalence, treatment costs and healthcare visits of patients with prostate cancer and bone metastasis.

Results A total of 1672 individuals were identified as having prostate cancer. Of these, 737 (44.1\%) had bone metastasis, and among these, this was already present at the time of initial prostate cancer diagnosis in 27.0\% (199/737). Mean age was 74.6 years $(S D \pm 9.1)$. Prevalence of prostate cancer with bone metastasis increased from 5.3 per 100000 males in 2011 to 8.3 per 100000 males in 2014. The total annual health expenditure per patient (in 2014 American dollars) during the study period was $\$ 15772.1$ ( $\mathrm{SD}=\$ 16$ 942.6) $\sim \$ 18206.3$ (SD=\$18 700.2); 88\% of these costs were reimbursed by insurance. Medication accounted for around $50 \%$ of total healthcare costs. Western drugs accounted for over $80 \%$ of medical costs with endocrine therapy being the most commonly prescribed treatment. There was an average $6.7 \%$ increase in expenditure related to diagnostical and therapeutical procedures over study years.

Conclusions The increase in the prevalence of prostate cancer with bone metastasis and associated healthcare costs in China reveals the growing clinical and economical burden of this disease. The high prevalence of bone metastasis among patients with prostate cancer seen in our study suggests that efforts may be needed to improve symptoms awareness and promote early help-seeking behaviour among the Chinese population.

\section{INTRODUCTION}

Prostate cancer is the most common cancer in males in the developed world and a leading cause of cancer deaths. ${ }^{1}$ In some less

\section{Strengths and limitations of this study}

- Our study is the first to estimate the prevalence of prostate cancer with bone metastasis in China.

- We used an administrative healthcare claims database of urban employees in Beijing in which the data were entered prospectively and therefore not subject to recall bias.

- The database included information from all cases of prostate cancer with bone metastasis treated in primary, secondary or tertiary hospitals therefore it is unlikely many cases will have been missed.

- Owing to the difference in age distribution between urban employees and the general male population of Beijing, prevalence estimates in this study could not be adjusted for age. Any future studies on this topic in populations with different age structures should bear this in mind if intending to make comparisons with our findings.

developed regions of the world - China, Brazil and Africa - incidence and mortality rates of prostate cancer have notably increased over recent decades. ${ }^{2-4}$ In China, national cancer registration data show that between 1998 and 2008, the incidence of prostate cancer increased at an annual rate of $12.1 \%$, and that in 2015, there were an estimated 60300 new cases of prostate cancer. ${ }^{3}$ This increase in incidence could be related to improved screening, ageing and changes in diet and other lifestyle factors. ${ }^{5}$

Prostate cancer will likely metastasise, especially to the bone, if not diagnosed and treated in the early stage of the disease. ${ }^{67}$ Bone metastasis are painful and can cause pathological bone fracture, spinal cord compression and reduced mobility, ${ }^{8}$ severely impacting on patients' quality of life ${ }^{9}$ and representing a substantial economical burden. ${ }^{11}{ }^{11}$ Globally, 
estimates of the prevalence of prostate cancer with bone metastasis are lacking. Published data are limited to a few studies reporting the proportion of prostate cancer cases with bone metastasis $-6.5 \%$ in the USA in $2004^{11}$ and $6.8 \%$ in Thailand between 2006 and $2015 .{ }^{12}$ Other data are limited to estimates of cumulative incidence of $11.5 \%$ in Denmark ${ }^{13}$ and $18 \%$ to $29 \%$ in the $\mathrm{USA}^{14}$ depending on follow-up duration.

After decades of effort and reform in the Chinese social health insurance system, medical insurance schemes are now well developed and administrative health databases have become a valuable resource for epidemiological and health economics research. The largest and most developed of these is the Urban Employee Basic Medical Insurance (UEBMI) claims database for employees in urban areas of China, which is administered by Beijing's medical insurance authorities. ${ }^{15} 16$ Using data from the UEBMI, we aimed to estimate the prevalence of all prostate cancer and of prostate cancer with bone metastasis, as well as associated direct medical costs among the male population of Beijing between 2011 and 2014.

\section{METHODS}

\section{Data source}

The UEBMI database holds information from visits (inpatient and outpatient to all public healthcare facilities including primary, secondary and tertiary hospitals) of both active and retired employees covered by the urban employee basic medical insurance scheme in Beijing. Over 14 million employees have participated in the scheme and, by the end of 2014, its coverage had reached over $98 \% .{ }^{17}$ No identifying personal information such as full name, citizen's ID number and contact information is held. In addition to medical information, the data also include demographics (including sex, age, the city of residence and type of insurance), and all direct expenditure information. Medical data includes the type of visit, the name of the hospital, level of the hospital (primary, secondary or tertiary), the name of the department, date of visit, principal diagnoses, secondary diagnoses, diagnostical procedures and prescribed drugs/therapeutical agents prescribed (western drugs are coded by internationally recognised Anatomical Therapeutic Chemical Classification System). Expenditure data include total medical expenditure, amount paid by the UEBMI insurance, method of medical insurance settlement, the unit price of service and quantity of service, subtotal expenditure (defined as the total expenditure for the service in a certain visit, ie, unit price of service times quantity of service) and the proportion of insurance payment (defined as the proportion of the amount paid by the insurer in total amount due).

\section{Study population and identification of prostate cancer patients}

We identified all patients in the UEBMI with prostate cancer between 1 January 2011 and 31 December
2014 as individuals with International Classification of Diseases-10 (ICD-10) code C61 (malignant neoplasm of prostate) plus a free text entry indicating prostate cancer during this period. We subsequently identified those with ICD-10 code C79.5 (metastatic carcinoma of bone) plus a free text entry indicating bone metastasis in prostate cancer either on or following the initial prostate cancer diagnosis. The index date was set as the date of the first record of bone metastasis. In addition, all patients were required to have at least 6 months continuous enrolment with the insurance scheme before the index date.

\section{Hospital visits and treatment costs}

For each patient with prostate cancer and bone metastasis we identified all records of hospital visits for any reasons (termed 'all visits'). As patients with prostate cancer may visit hospital for reasons other than prostate cancer, visits directly related to the diagnosis or treatment of prostate cancer with bone metastasis were called 'valid visits'. These were identified from entries for ICD-10 codes C61 and C79.5 together with associated free text entries for prostate cancer and for bone metastasis in prostate cancer. We calculated annual medical care costs per hospital visit as well as per capita stratified by visit type (inpatient vs outpatient) and patient setting. The percentage of medical costs covered by UEBMI was also calculated. A separate cost analysis was conducted for total costs per capita. Total costs included all items eligible for reimbursement: drugs and diagnostical/therapeutical procedures. Medications were divided into western and Traditional Chinese Medicines (TCM). Western drugs were divided into four categories: radiotherapy drugs, chemotherapy drugs, hormone therapy drugs and bisphosphonates. Diagnostical and therapeutical procedures evaluated included examination, surgery, radiotherapy, inpatient stay, nursing care, medical device and other diagnostical fees. Traditional Chinese drugs were classified according to the Chinese Urological Association guideline (2014) for the treatment of prostate cancer. ${ }^{18}$ We calculated the constituent ratio of both drugs and diagnostical/therapeutical items based on all visits. Costs in US dollars were calculated using the medical care component of the Consumer Price Index in China on December $2014^{19}$ based on the average conversion rate of $6.1 ¥ / \mathrm{US} \$$ in $2014 .^{20}$

\section{Statistical analyses}

We calculated the prevalence of prostate cancer (with or without metastasis) on 31 December of each study year by dividing the number of patients with prostate cancer and bone metastasis by the total number of males in the database at this time point, and expressed per 100000 males. The denominator for the prevalence calculations was obtained from the annual government official report and statistical yearbook for the total population of male employees participating in the UEBMI. For hospital visits and treatment costs, data were described using frequencies and percentages for categorical variables, and means with SD and medians with IQR for continuous variables. 
Table 1 Prevalence of prostate cancer with bone metastasis in Beijing from 2011 to 2014

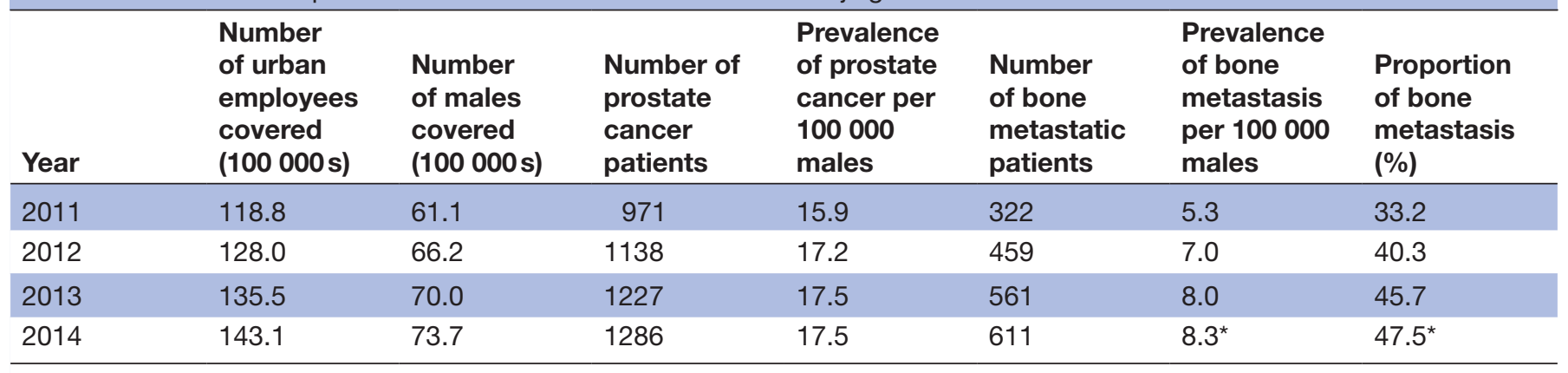

${ }^{*} \mathrm{P}$ value $<0.001$ for change over the study period.

The Student's t-test was applied to compare differences between groups for continuous variables. Analyses were conducted using SAS statistical software (V.9.2).

\section{Patient and public involvement}

There was no public or patient involvement in the conception of the research question or the design or implementation of the study.

\section{RESULTS}

A total of 1672 individuals were identified as having prostate cancer between 2011 and 2014. Of these, 737 (44.1\%) had bone metastasis, - this was already present at the time of initial prostate cancer diagnosis in 27\% (199/737). The mean age was 74.6 years (SD 9.1), and median age was 76 years (range 30 to 95 years). Three quarters of the patients were aged 70 years or more at first diagnosis of bone metastasis.

\section{Prevalence}

Table 1 shows the prevalence of prostate cancer with bone metastasis, as well as the proportion of all patients with prostate cancer who had bone metastasis in each study year. The prevalence of all prostate cancer was 15.9 per 100000 males in 2011 rising to 17.5 per 100000 males in 2014. The prevalence of prostate cancer with bone metastasis was 5.3 per 100000 males in 2011, rising in each study year to 8.3 per 100000 males in $2014(\mathrm{p}<0.001)$. Patients with bone metastases accounted for $33.2 \%$ of prostate cancer patients in 2011 increasing to $47.5 \%$ in $2014(\mathrm{p}<0.001)$.

\section{Healthcare visits}

Visits to medical institutions are shown in table 2. The total number of valid visits and all visits for the 4 years were 31353 and 108 807, respectively. Eighty-nine per cent of valid visits were to tertiary hospitals compared with only $59.9 \%$ of all visits. The majority of hospital visits, both valid visits and all visits, were on an outpatient basis. However, the percentage of valid visits among all visits during the study period was three times higher for inpatient visits $(75.1 \%)$ than for outpatient visits $(25.0 \%)$. On average, patients had at least three hospital visits and 10 outpatient visits per year due to their bone metastasis.

\section{Treatment costs}

As shown in table 3, total, inpatient and outpatient costs among patients with prostate cancer and bone metastasis based on all visits increased across the study period. The mean cost of outpatient visits per capita rose from $\$ 5503.3$ (SD \$6137.7) in 2011 to $\$ 6844.8$ (SD \$6829.1) in 2014, while the mean cost of inpatient visits rose from $\$ 12726.8$ (SD \$13 469.9) to \$14218.6 (SD \$14 890.7) across study years. The percentage of outpatient and inpatient

Table 2 Distribution of hospital visits among patients with prostate cancer and bone metastasis

\begin{tabular}{lcc}
\hline & Valid visits* (n, \%) & All visits (n, \%) \\
\hline $\begin{array}{l}\text { Medical institution } \\
\text { Tertiary hospital }\end{array}$ & $27997(89.3)$ & $65183(59.9)$ \\
$\begin{array}{l}\text { Secondary } \\
\text { hospital }\end{array}$ & $2669(8.5)$ & $14518(13.3)$ \\
\hline Primary hospital & $687(2.2)$ & $29106(26.8)$ \\
\hline Total & $31353(100.0)$ & $108807(100.0)$ \\
Visits, time & & \\
\hline Outpatient & & \\
\hline 2011 & $3298(13.1)$ & $13025(13.0)$ \\
2012 & $6112(24.3)$ & $24193(24.1)$ \\
2013 & $7612(30.3)$ & $31121(30.9)$ \\
2014 & $8131(32.3)$ & $32215(32.0)$ \\
\hline Total & $25153(100.0)$ & $100554(100.0)$ \\
\hline Inpatient & & \\
2011 & $908(14.6)$ & $1140(13.8)$ \\
2012 & $1417(22.9)$ & $1912(23.2)$ \\
2013 & $1797(29.0)$ & $2432(29.5)$ \\
2014 & $2078(33.5)$ & $2769(33.5)$ \\
\hline Total & $6200(100.0)$ & $8253(100.0)$ \\
\hline
\end{tabular}

*Visits directly related to the diagnosis or treatment of prostate cancer with bone metastasis. 
Table 3 Total expenditure and reimbursement percentages for patients with prostate cancer and bone metastasis in Beijing from 2011 to 2014 (per patient per year)

\begin{tabular}{|c|c|c|c|c|c|c|c|c|}
\hline Year & Setting & $\mathbf{N}$ & Mean & SD & Median & Q1 & Q3 & $\% †$ \\
\hline \multirow[t]{3}{*}{2011} & Outpatient & 310 & 5503.3 & 6137.7 & 3416.0 & 1609.6 & 6773.7 & $93.0 \%$ \\
\hline & Inpatient & 265 & 12726.8 & 13469.9 & 7536.3 & 3322.8 & 17701.0 & $87.4 \%$ \\
\hline & Total & 322 & 15772.1 & 16942.6 & 9360.2 & 4328.9 & 21147.6 & $89.3 \%$ \\
\hline \multirow[t]{3}{*}{2012} & Outpatient & 447 & 6959.0 & 6976.3 & 4870.7 & 2389.5 & 8975.7 & $93.1 \%$ \\
\hline & Inpatient & 390 & 13745.0 & 15243.1 & 8551.3 & 3507.1 & 18323.3 & $88.0 \%$ \\
\hline & Total & 458 & 18496.2 & 18931.6 & 11704.7 & 5974.4 & 24070.1 & $89.9 \%$ \\
\hline \multirow[t]{3}{*}{2013} & Outpatient & 552 & 6992.4 & 6990.6 & 4454.3 & 2137.9 & 8897.5 & $93.0 \%$ \\
\hline & Inpatient & 451 & 13555.3 & 14713.5 & 8411.0 & 3381.9 & 18909.8 & $87.9 \%$ \\
\hline & Total & 558 & 17873.3 & 18562.8 & 11144.6 & 4789.1 & 24630.8 & $89.9 \%$ \\
\hline \multirow[t]{3}{*}{2014} & Outpatient & 599 & 6844.8 & 6829.1 & 4258.4 & 2439.7 & 8854.6 & $92.8 \%$ \\
\hline & Inpatient & 494 & 14218.6 & 14890.7 & 9529.8 & 4431.4 & 19005.0 & $86.0 \%$ \\
\hline & Total & 611 & 18206.3 & 18700.2 & 11801.0 & 5612.8 & 24711.6 & $88.5 \%$ \\
\hline
\end{tabular}

${ }^{*}$ Based on all visits. Q1 = 25\% quartile/Q3 = 75\% quartile.

†The proportion of treatment costs reimbursed by Urban Employee Basic Medical Insurance. SD, standard deviation.

treatment costs reimbursed by UEBMI during the study period was approximately $93 \%$ and $88 \%$, respectively.

Among patients treated during our observation period, medications accounted for around half of total costs, with diagnostical and therapeutical procedures accounting for the other half. As shown in table 4, western drugs cost accounted for more than $80 \%$ of total medication costs, of which endocrine therapy drugs were the most frequently prescribed. Traditional Chinese drugs were used by most patients, while radiotherapy and chemotherapy were less frequently administered. Costs related to diagnostical and therapeutical procedures are shown in table 5. For each study year, over $98 \%$ of patients had at least one clinical examination. The mean costs of diagnostical and therapeutical procedures increased to $\$ 8858.0$ in 2014 (a rise of $6.7 \%$ from 2011). Expenditures for examinations and medical devices per capita increased significantly over the study period while the annual output for radiotherapy and nursing care decreased $(\mathrm{p}<0.001)$.

\section{DISCUSSION}

Our study has revealed the growing burden of prostate cancer with bone metastasis in China. Using data from a large administrative claims database, we found that between 2011 and 2014, the prevalence of prostate cancer with bone metastasis among male urban employees of Beijing increased from 5.3 per 100000 males to 8.3 per 100000 males with a parallel increase in associated healthcare costs.

The main strength of our study is its novelty. We are unaware of other estimates of the prevalence of prostate cancer with bone metastasis worldwide, or of associated healthcare costs in China. Another strength is the large population-based data source representative of male urban employees in Beijing and including information on all healthcare visits whether to primary, secondary or tertiary institutions (where, in China, nearly all cancers are treated).$^{21}$ Our operational case definition required both a diagnosis of prostate cancer and of bone metastasis in prostate cancer (either concurrently or subsequently) together with free text entries indicative of both to minimise the number of false positives. However, we cannot exclude the possibility that some true cases may have been missed due to under-recording of relevant ICD-10 codes and/or the absence of free text entries, which could have led to underestimated prevalence estimates. A limitation of our study is that we were only able to calculate crude estimates of prostate cancer prevalence and not age-adjusted estimates because information on the age distribution of urban employees could not be obtained. Owing to the difference in age distribution between urban employees and the general population, our prevalence estimates cannot be generalised to all Chinese males, and all our study's findings are only generalisable to male employees in the city of Beijing. Any future studies on this topic in populations with different age structures should also bear this in mind if intending to make comparisons with our findings. Another limitation is that because date of death is not recorded in the UEBMI and the database is not linked to the city's death registry, we were unable to exclude patients who had died before 31 December in each study year in our prevalence calculations, and which would have led to some degree of underestimated prevalence estimates.

The proportion of patients with prostate cancer in our study who had bone metastasis $-33.2 \%$ in 2011 , rising to $47.5 \%$ in 2014 - is much higher than reports from the USA $(6.5 \%)^{11}$ in the previous decade (2004) and from Thailand between 2006 and $2015 .{ }^{12}$ This could be explained by recent advances in diagnostical methods, including the enhanced sensitivity of diagnostical tools enabling higher case detection, and developments in surgery, 


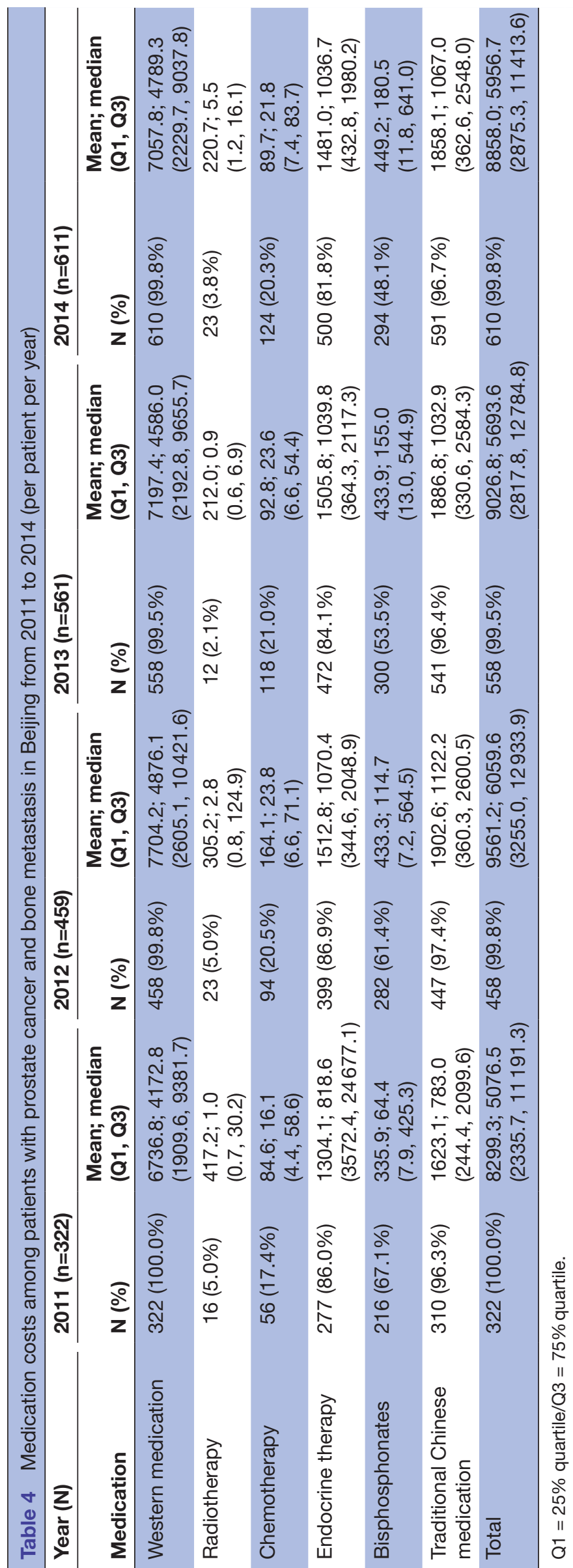




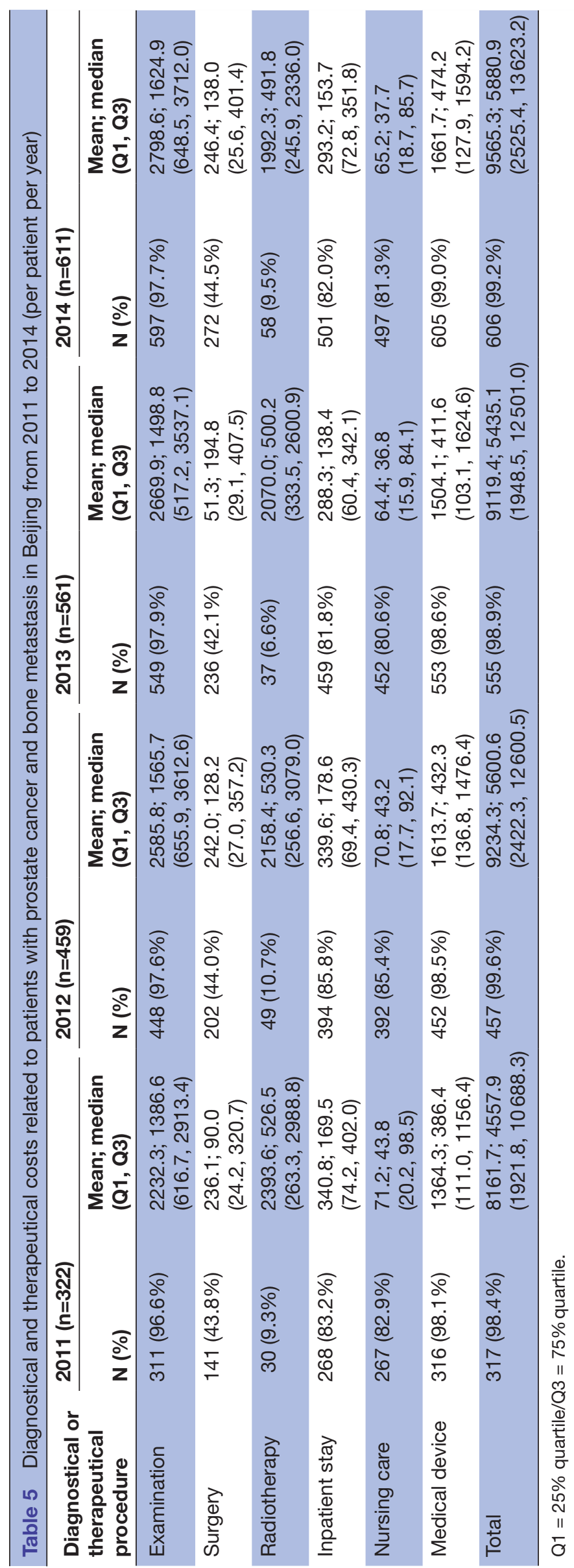


radiotherapy, chemotherapy and other supportive treatments, leading to increased survival. ${ }^{22}$ Also, we had access to all medical visits occurring in primary, secondary or tertiary hospitals, thus having maximum opportunity to capture all cases of bone metastasis. Another possible reason could be lower awareness of prostate cancer symptoms among the Chinese population compared with other countries, or other reasons for delay in helpseeking, with medical attention often only sought after the development of serious, painful symptoms or bone fractures - among patients with bone metastasis in our study, over a quarter had their diagnosis of bone metastasis at the time of initial prostate cancer diagnosis.

Costs per capita for inpatient visits were consistently around double those for outpatient visits across the study period. Drug expenditure accounted for $68 \%$ of the total expenditure among these patients, and unsurprisingly was highest for hormone therapy - a known effective treatment in this patient population. ${ }^{1832}{ }^{24}$ In China, TCM is widely used as adjuvant therapy in cancer treatment, and this was clearly shown in our study, where over $95 \%$ of patients received TCM, accounting for one-fifth of total treatment costs. The majority of visits (89\%) among patient with prostate cancer (with or without bone metastasis) in our study were to tertiary hospitals. This might be explained by the status of unbalanced medical resource in China. Also, the value that patients give to the advantages of tertiary hospitals - specialised doctors, and better diagnostics and treatment options - may override any concerns over costs.

As the population continues to age and more sophisticated diagnostical and treatment methods are more widely implemented, China will likely see an increased prevalence of patients with prostate cancer and bone metastasis, although future studies will be needed to investigate this. The increasing clinical and economical burden will be an important knowledge for healthcare decision makers in the country. The higher proportion of patients with prostate cancer who have bone metastasis in this study (compared with other countries) suggests the need for public health awareness regarding symptom development and efforts to improve early help-seeking.

\author{
Author affiliations \\ ${ }^{1}$ Department of Epidemiology and Biostatistics, School of Public Health, Peking \\ University, Beijing, China \\ ${ }^{2}$ Department of Pharmacy, Peking University Third Hospital, Beijing, China \\ ${ }^{3}$ Epidemiology, Bayer U.S. Whippany, New Jersey, USA \\ ${ }^{4} \mathrm{HEOR}$ and Medical Affairs, Bayer Healthcare Co., Ltd., Beijing, China \\ ${ }^{5}$ Epidemiology, Bayer AG, Berlin, Germany \\ ${ }^{6}$ Peking University First Hospital, Beijing, China \\ ${ }^{7}$ Department of Urology, Peking University Third Hospital, Beijing, China
}

Acknowledgements We thank EpiMed Communications Ltd (Oxford, UK) for medical writing assistance funded by Bayer AG and Shuangqing Gao for support with data acquisition.

Contributors LZ performed the statistical analysis and wrote the manuscript. LZ, YC, YP and SZ contributed to the study design, interpretation of the data and the discussion of the manuscript. JZ, WS, LX, MS, YS and JL contributed to the manuscript revise and provided clinical support. All the authors supplied critical revisions to the manuscript and gave approval of the final version of the manuscript to be published.

Funding This work was supported by the National Natural Science Foundation of China (91646107) and Bayer AG, Berlin, Germany (89300-4432 101 080).

Competing interests We have read and understood BMJ policy on declaration of interests and declare the following interests: Jihong Zong is an employee of Epidemiology, Bayer US. WentaoSun and Lin Xu are employees of HEOR and Medical Affairs, Bayer Healthcare Co, Ltd. Montse Soriano-Gabarró is an employee of Epidemiology, Bayer AG, Berlin, Germany. All other authors report no potential conflict of interest.

Patient consent for publication Not required.

Provenance and peer review Not commissioned; externally peer reviewed.

Data sharing statement Extracted data that support the findings of this study are available from Beijing Municipal Health Insurance Bureau but restrictions apply to the availability of these data, which were used under license for the current study, and so are not publicly available. Data are however available from the authors upon reasonable request and with permission of Beijing Municipal Health Insurance Bureau.

Open access This is an open access article distributed in accordance with the Creative Commons Attribution Non Commercial (CC BY-NC 4.0) license, which permits others to distribute, remix, adapt, build upon this work non-commercially, and license their derivative works on different terms, provided the original work is properly cited, appropriate credit is given, any changes made indicated, and the use is non-commercial. See: http://creativecommons.org/licenses/by-nc/4.0/.

\section{REFERENCES}

1. Bray F, Ferlay J, Soerjomataram I, et al. Global cancer statistics 2018: GLOBOCAN estimates of incidence and mortality worldwide for 36 cancers in 185 countries. CA Cancer J Clin 2018;68:394-424.

2. Chatenoud L, Bertuccio P, Bosetti C, et al. Trends in cancer mortality in Brazil, 1980-2004. Eur J Cancer Prev 2010;19:79-86.

3. Chen W, Zheng R, Baade PD, et al. Cancer statistics in China, 2015. CA Cancer J Clin 2016;66:115-32.

4. Parkin DM, Bray F, Ferlay J, et al. Cancer in africa 2012. Cancer Epidemiology and Prevention 2014.

5. Chen R, Ren S, Yiu MK, et al. Prostate cancer in Asia: A collaborative report. Asian J Urol 2014;1:15-29.

6. Briganti A, Suardi N, Gallina A, et al. Predicting the risk of bone metastasis in prostate cancer. Cancer Treat Rev 2014;40:3-11.

7. Chien TM, Lu YM, Geng JH, et al. Predictors of Positive Bone Metastasis in Newly Diagnosed Prostate Cancer Patients. Asian Pac J Cancer Prev 2016;17:1187-91.

8. Saad F, Clarke N, Colombel M. Natural history and treatment of bone complications in prostate cancer. Eur Urol 2006;49:429-40.

9. Thobe MN, Clark RJ, Bainer RO, et al. From prostate to bone: key players in prostate cancer bone metastasis. Cancers 2011;3:478-93.

10. Yong $\mathrm{C}$, Onukwugha E, Mullins CD. Clinical and economic burden of bone metastasis and skeletal-related events in prostate cancer. Curr Opin Oncol 2014;26:274-83.

11. Schulman KL, Kohles J. Economic burden of metastatic bone disease in the U.S. Cancer 2007;109:2334-42.

12. Phanphaisarn A, Patumanond J, Settakorn J, et al. Prevalence and Survival Patterns of Patients with Bone Metastasis from Common Cancers in Thailand. Asian Pac J Cancer Prev 2016;17:4335-40.

13. Nørgaard M, Jensen $A \varnothing$, Jacobsen JB, et al. Skeletal related events, bone metastasis and survival of prostate cancer: a population based cohort study in Denmark (1999 to 2007). J Urol 2010;184:162-7.

14. Hernandez RK, Wade SW, Reich A, et al. Incidence of bone metastases in patients with solid tumors: analysis of oncology electronic medical records in the United States. BMC Cancer 2018;18:44.

15. Li X, Lu J, Hu S, et al. The primary health-care system in China. The Lancet 2017;390:2584-94.

16. Sun Y, Gregersen H, Yuan W. Chinese health care system and clinical epidemiology. Clin Epidemiol 2017;9:167-78.

17. Beijing Municipal Human Resources and Social Security Bureau. 2015. Beijing social security develpment report. http://www.bjrbj.gov. cn/xxgk/gsgg/201601/t20160104_55657.html

18. Urological oncology professional committee of Chinese anti-cancer association. China castration-resistant prostate cancer diagnosis and treatment experts consensus. Chinese journal of surgery 2016;54:481-4. 
19. National Bureau of Statistics of the People's Republic of China. Chinese statistics yearbook 2014. Beijing: China Statistics Press, 2014:1-937.

20. The People's Bank of China. Foreign Exchange Quotation. 2018 http://www.pbc.gov.cn/rmyh/108976/109428/index.html (accessed 06 May 2018).

21. Gu W, Xu YM, Zhong BL. Health-related quality of life in Chinese inpatients with lung cancer treatedin large general hospitals: acrosssectional study. BMJ Open 2018;8:e019873.
22. Berg KD, Thomsen FB, Mikkelsen MK, et al. Improved survival for patients with de novo metastatic prostate cancer in the last 20 years. Eur J Cancer 2017;72:20-7.

23. Bourke L, Kirkbride P, Hooper R, et al. Endocrine therapy in prostate cancer: time for reappraisal of risks. benefits and cost-effectiveness? British journal of cancer 2013;108:9.

24. Sweeney CJ, Chen YH, Carducci M, et al. Chemohormonal Therapy in Metastatic Hormone-Sensitive Prostate Cancer. N Engl J Med 2015;373:737-46. 